\title{
Response of a Hodgkin-Huxley neuron to a high-frequency input
}

\author{
L. S. Borkowski \\ Faculty of Physics, Adam Mickiewicz University, Umultowska 85, 61-614 Poznan, Poland
}

\begin{abstract}
We study the response of a Hodgkin-Huxley neuron stimulated by a periodic sequence of conductance pulses arriving through the synapse in the high frequency regime. In addition to the usual excitation threshold there is a smooth crossover from the firing to the silent regime for increasing pulse amplitude $g_{s y n}$. The amplitude of the voltage spikes decreases approximately linearly with $g_{\text {syn }}$.

In some regions of parameter space the response is irregular, probably chaotic. In the chaotic regime between the mode-locked regions 3:1 and 2:1 near the lower excitation threshold the output interspike interval histogram (ISIH) undergoes a sharp transition. If the driving period is below the critical value, $T_{i}<T^{*}$, the output histogram contains only odd multiples of $T_{i}$. For $T_{i}>T^{*}$ even multiples of $T_{i}$ also appear in the histogram, starting from the largest values. Near $T^{*}$ the ISIH scales logarithmically on both sides of the transition. The coefficient of variation of ISIH has a cusp singularity at $T^{*}$. The average response period has a maximum slightly above $T^{*}$. Near the excitation threshold in the chaotic regime the average firing rate rises sublinearly from frequencies of order $1 \mathrm{~Hz}$.
\end{abstract}

\section{INTRODUCTION}

Biological neurons transmit information in the form of sharp spikes of potential difference across the lipid bilayer forming the wall of the nerve cell. This feature of the cell's reaction to input signals is remarkably consistent in different organisms and different types of neurons. The action potential spikes are assumed to be the principal carrier of information. The early view that information is transmitted via rate coding has evolved. It is now recognized that also the spike time coding is used in neural systems 1.2 . While the precise coding recipe is unknown it is clear that the knowledge of the response of various types of neurons to different stimuli is fundamental to formulating the theory of information transfer in the neural system.

Our understanding of conductance-based models of neurons is largely based on the Hodgkin-Huxley (HH) model originally formulated to describe the dynamics of the membrane potential of the squid giant axon ${ }^{3}$. The detailed voltage-clamp measurements of the voltage-gated potassium and sodium ion currents led to revisions of the $\mathrm{HH}$ model. The modifications required to achieve better agreement with experiments were reviewed by Clay 4 . Studies of single neurons and neuronal networks often employ simplified models, such as integrate-and-fire and FitzHugh-Nagumo (FHN) models 5.6 . It is believed that the two-dimensional flow models such as FHN reproduce qualitatively the behavior of the $\mathrm{HH}$ model. However these simplifications are not always justifiable $e^{\underline{7}-\underline{\underline{9}}}$. In an interesting analysis of chaos in the HH model Guckenheimer and Oliva ${ }^{9}$ point out that even the concept of a firing threshold may be more subtle that just a smooth hypersurface dividing subthreshold and suprathreshold membrane potentials.

Over the years many studies of $\mathrm{HH}$ equations were carried out, including stochastic variations of various quantities $\frac{10-12}{-12}$. An important question is to what extent the qualitative properties of neuron response depend on the functional form of the input signal. One frequently used form of input is constant plus a sinusoidal term. However the physiological signals are more pulse-like. In a strongly nonlinear system this may lead to substantial differences in the output.

In the sinusoidally driven $\mathrm{HH}$ model the excitation threshold rises sharply at large frequencies. The phase diagram in the frequency-current amplitude plane consists of three phase locked regions with integer ratio of the output period to the input period, $\bar{T}_{o} / T_{i}, 1: 1,2: 1$, and $3: 1$. There are also areas of fractional locking and bistable or chaotic response around these phase-locked states $\underline{13}-15$.

It was pointed out that the edges of mode-locked plateaus have analogies to phase transitions in the equilibrium statistical mechanics. Two forms of scaling of the average deviation from perfect mode-locking were found near the edges of plateaus with constant $p / q$, where $p$ and $q$ are integers, indicating number of input spikes per number of output action potentials $\frac{16}{}$. The scaling has either exponent $1 / 2$ or is logarithmic. In this paper we will show that scaling is more common and appears also near the multimodal transition points.

Here we assume the $\alpha$ form of postsynaptic current, $I_{\text {syn }} \sim t \exp (-t / \tau)$, where $t$ is time from the onset of the input spike and $\tau$ is the time scale of the synaptic action. This form is close to experimental observation although it does not take into account a more complex dynamics of the ion channel kinetics, usually described in the Markovian scheme.

The general form of the phase diagram of the HodgkinHuxley model with this input was studied initially in Ref ${ }^{17}$. However many important questions are still to be answered. One of them is the behavior of the system in the high-frequency limit. In the following we present the model and show the main features of high-frequency response. 


\section{THE MODEL}

The Hodgkin-Huxley neuron subject to periodic conductance pulses is defined by the following set of equations,,$\frac{3}{,}$

$$
\begin{gathered}
C d V / d t=-g_{N a} m^{3} h\left(V-V_{N a}\right)-g_{K} n^{4}\left(V-V_{K}\right) \\
-g_{L}\left(V-V_{L}\right)+I_{e x t}+I_{s y n}, \\
d m / d t=-\left(a_{m}+b_{m}\right) m+a_{m}, \\
d h / d t=-\left(a_{h}+b_{h}\right) h+a_{h}, \\
d n / d t=-\left(a_{n}+b_{n}\right) n+a_{n}, \\
\end{gathered}
$$

where

$$
\begin{gathered}
a_{m}=0.1(V+40) /\left[1-e^{-(V+40) / 10}\right], \\
b_{m}=4 e^{-(V+65) / 18}, \\
a_{h}=0.07 e^{-(V+65) / 20}, \\
b_{h}=1 /\left[1+e^{-(V+35) / 10}\right], \\
a_{n}=0.01(V+55) /\left[1-e^{-(V+55) / 10}\right], \\
b_{n}=0.125 e^{-(V+65) / 80} .
\end{gathered}
$$

In equations (5)-(10) the voltage is expressed in $\mathrm{mV}$ and the rate constants $\alpha$ and $\beta$ are given in $\mathrm{ms}^{-1}$. The reversal potentials of sodium, potasium and leakage channels are $V_{N a}=50 \mathrm{mV}, V_{K}=-77 \mathrm{mV}$, and $V_{L}=-54.5 \mathrm{mV}$, respectively. The corresponding maximum conductances are $g_{N a}=50 \mathrm{mV}, g_{K}=36 \mathrm{mS} / \mathrm{cm}^{2}$, and $g_{L}=0.3 \mathrm{mS} / \mathrm{cm}^{2}$. The capacity of the membrane is $C=1 \mu \mathrm{F} / \mathrm{cm}^{2} \underline{\underline{3}}$.

The synaptic current $I_{s y n}$ is given by the following equation,

$$
I_{\text {syn }}(t)=g_{\text {syn }} \sum_{n} \alpha\left(t-t_{i n}\right)\left(V_{a}-V_{s y n}\right),
$$

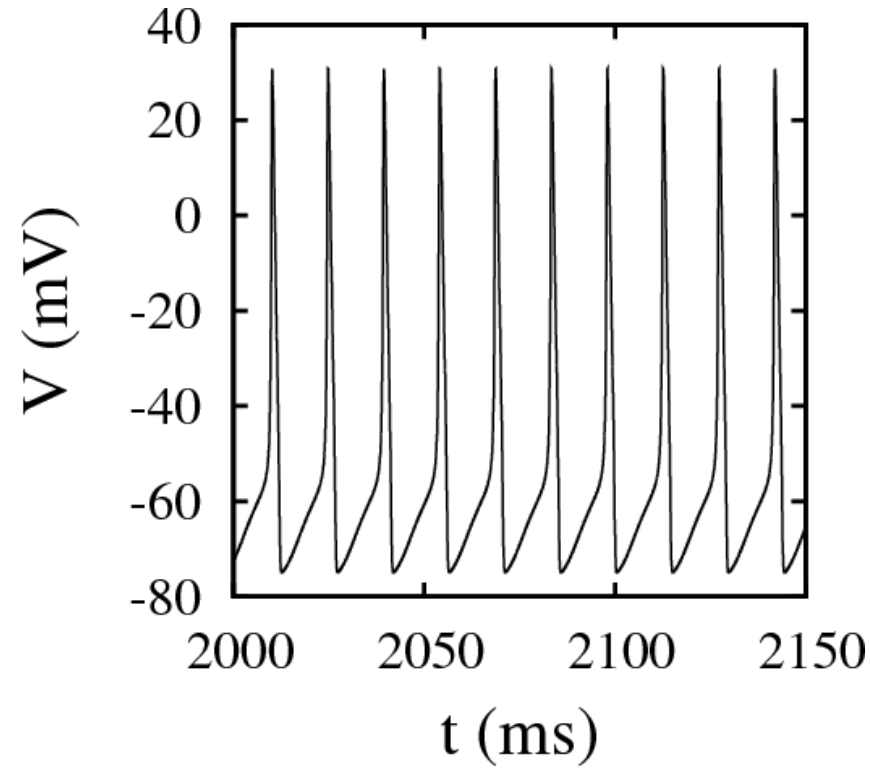

FIG. 1: Sample voltage trace for a constant input current $I_{\text {ext }}=10 \mu \mathrm{A} / \mathrm{cm}^{2}$.

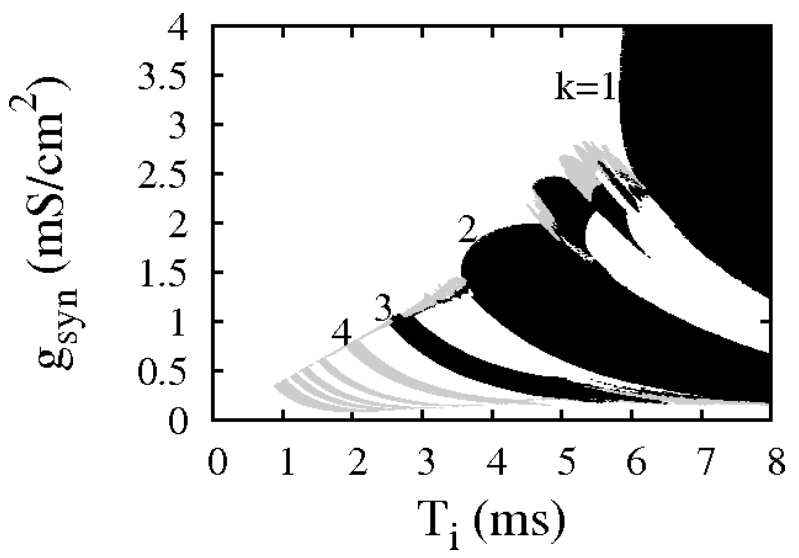

FIG. 2: The ratio of the average output spiking rate to the input rate, $k=\bar{T}_{o} / T_{i}$. Mode-locked regions with $k=1,2,3$ and $k=4,5,6,7,8$ are shown in black and grey respectively. Voltage peaks were counted as spikes when $V$ exceeded 0 . For high values of $g_{s y n}$ the neuron does not respond.

where $t_{i n}$ denotes the start of the $n^{\text {th }}$ pulse, $g_{\text {syn }}$ is the conductivity of the synapse, $V_{a}=30 \mathrm{mV}$ is the maximum potential in the postsynaptic area and $V_{\text {syn }}=-50 \mathrm{mV}$ is the reversal potential of the synapse. The period of the synaptic drive is $T_{i}=t_{i n+1}-t_{i n}$. The external current $I_{\text {ext }}$ is set to 0, except for a sample run shown in Fig. 1.

The time-dependence is given by the function

$$
\alpha(t)=(t / \tau) e^{-t / \tau)} \Theta(t)
$$

where $\tau$ is time scale characterizing the dynamics of the synaptic action and $\Theta(t)$ is the Heaviside step function. We study the dependence of the output interspike separation $T_{o}$ on $T_{i}$ and $g_{s y n}$. 

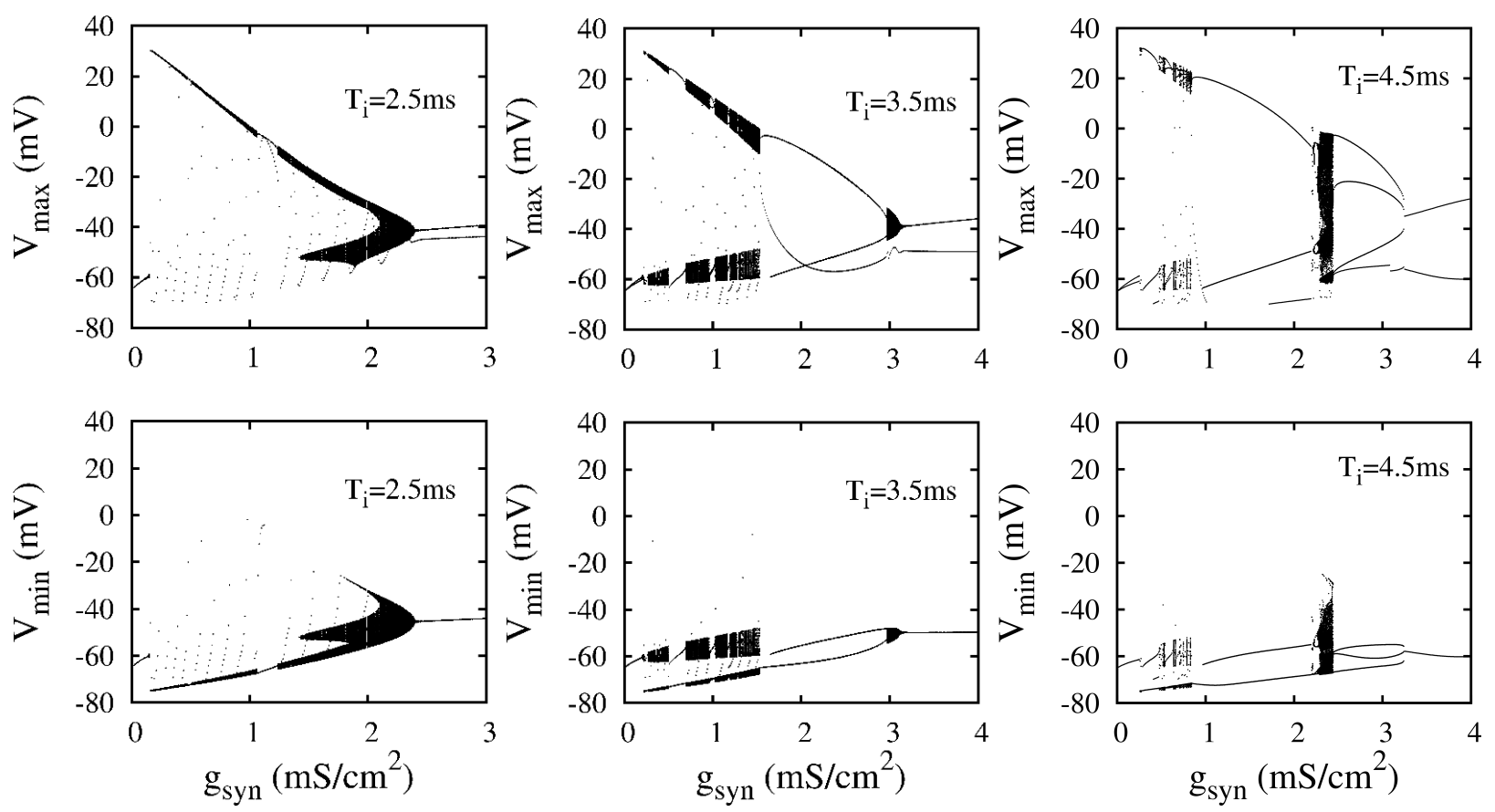

FIG. 3: Values of maxima and minima of the membrane potential $V(t)$ as a function of synaptic conductivity $g_{s y n}$ for input spike intervals $T_{i}=2.5 \mathrm{~ms}, 3.5 \mathrm{~ms}$, and $4.5 \mathrm{~ms}$.

Equations (11)-(10) were integrated with the fourth order Runge-Kutta scheme. The time step was $0.01 \mathrm{~ms}$. For each parameter set the simulation was run for 30 seconds. Results of the initial three seconds of each data set were discarded to avoid transient behavior. In the chaotic regime the data were obtained from five runs for each value of the horizontal coordinate.

\section{RESULTS}

The average output spiking rate in the form of a color map as a function of the input period $T_{i}$ and maximum synaptic conductivity $g_{\text {syn }}$ is presented in Fig. 2. The mode-locked regions are shown as areas of uniform color. For small $T_{i}$ the total incoming current is approximately constant with a small modulation, and the excitation threshold rises linearly with increasing $T_{i}$, $g_{\text {syn }} \simeq 0.04 T_{i} \mathrm{mS} /\left(\mathrm{ms} \mathrm{cm}^{2}\right)$. For $g_{\text {syn }}$ exceeding approximately $0.4 T_{i} \mathrm{mS} /\left(\mathrm{ms} \mathrm{cm}^{2}\right)$ the spiking action does not occur. We can see from Fig. 2 that this behavior sets in below $T_{i} \simeq 6 \mathrm{~ms}$.

The obtained phase diagram is qualitatively different from a response to a sinusoidal input, where the excitation threshold diverges as $1 / T_{i}$, for $T_{i} \rightarrow 0$. In general we may expect that the constraint of charge balancing, $\int_{t}^{t+T_{i}} I d t=0$, will have a significant impact at high frequencies. For intermediate values of the input period, $5 \mathrm{~ms}<T_{i}<13 \mathrm{~ms}$, the topology of the phase diagram resembles results obtained with sinusoidal input, see e.g. Fig. 2 of Ref $\frac{13}{2}$.

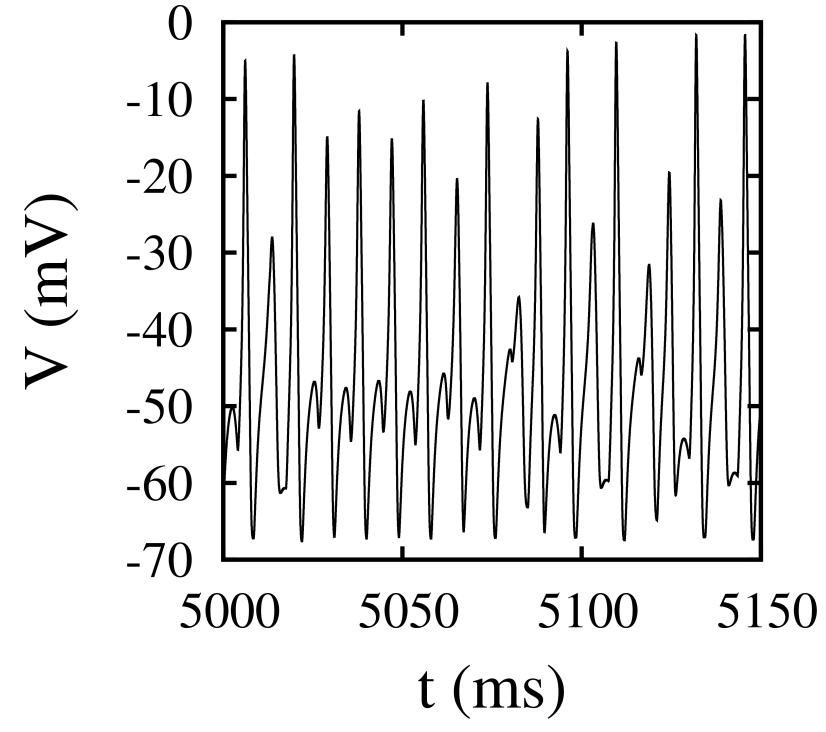

FIG. 4: For high synaptic conductivities the distinction between action potential and the background oscillations loses its meaning. This sample was obtained for $T_{i}=4.5 \mathrm{~ms}$ and $g_{\text {syn }}=2.35 \mathrm{mS} / \mathrm{cm}^{2}$.

Fig. 3 shows dependence of minima and maxima of $V$ on $g_{\text {syn }}$ for three input frequencies. The amplitude of response decreases linearly with increasing $g_{\text {syn }}$. There is no well-defined spiking threshold. There are intervals of parameter values for which the response is highly irregular and the values of maxima and minima of $V$ vary significantly. 

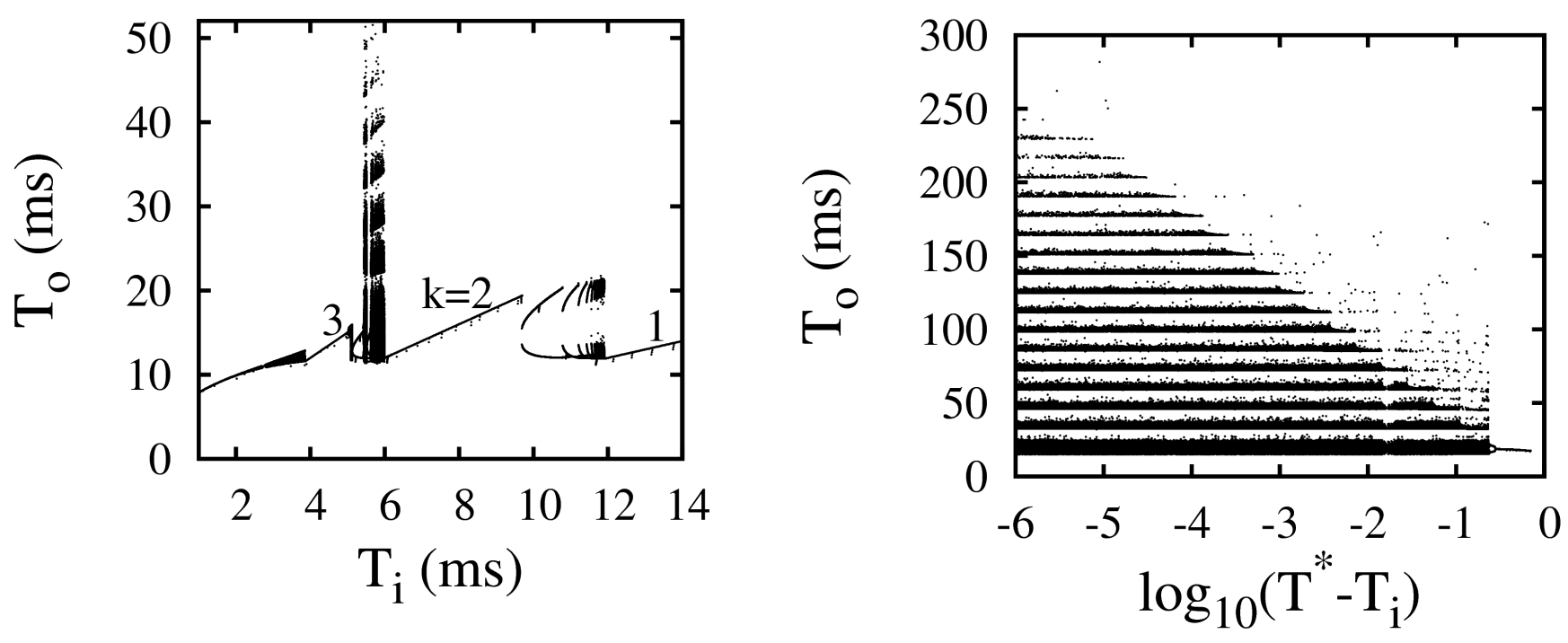

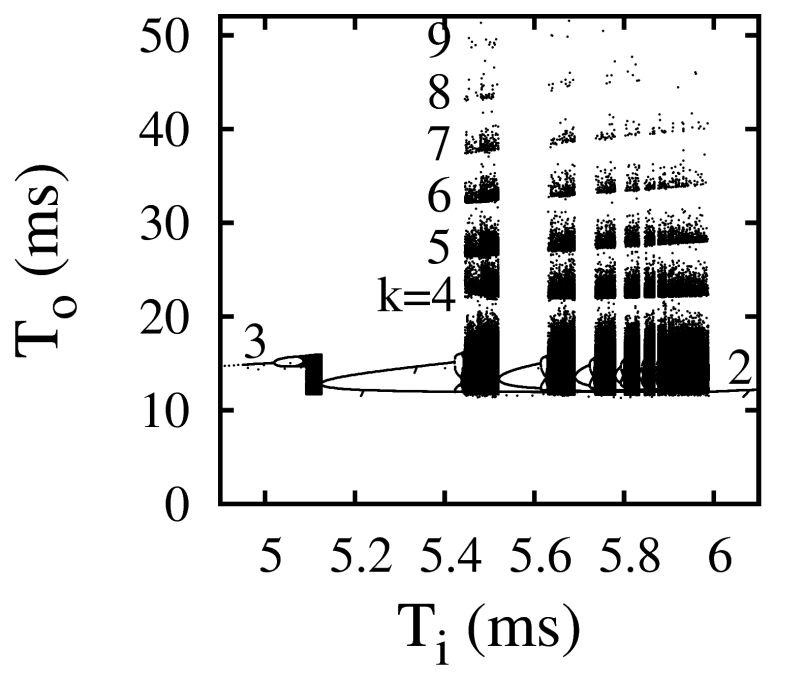

FIG. 5: (a)The spectrum of interspike separations of the output signal as a function of the input period $T_{i}$ for $g_{\text {syn }}=$ $0.4 \mathrm{mS} / \mathrm{cm}^{2}$, (b) Detailed view of the chaotic region between $T_{i}=5 \mathrm{~ms}$ and $6 \mathrm{~ms}$. Each ISI cluster belongs to different $k$, where $k=2,3,4,5, \ldots$. The distinction between $k=2$ and $k=3$ is blurred.

A sample time-dependence of the membrane potential is shown in Fig. 4. The maxima of $V$ span almost the entire range between $-60 \mathrm{mV}$ and $0 \mathrm{mV}$. There is no clear separation of spikes from the rest of the signal.

Chaotic behavior in the parameter space between the 3:1 and 2:1 mode-locked regions leads to multimodal response. The interspike separation for $g_{\text {syn }}=0.4 \mathrm{mS} / \mathrm{cm}^{2}$ is shown in Fig. 5. For $T_{i}$ between $5.5 \mathrm{~ms}$ and $6 \mathrm{~ms}$ all integer multiples of input $T_{i}$ with the exception of the lowest one appear in the output ISIH.

It is interesting to note that ISI histograms (ISIH) from some older experiments on nerve fibers of monkeys $\frac{18}{\underline{1}}$ and single neurons in the primary visual cortex of a cat $\underline{\underline{19}}$ show some similarity to Fig. [5. Experimental histograms

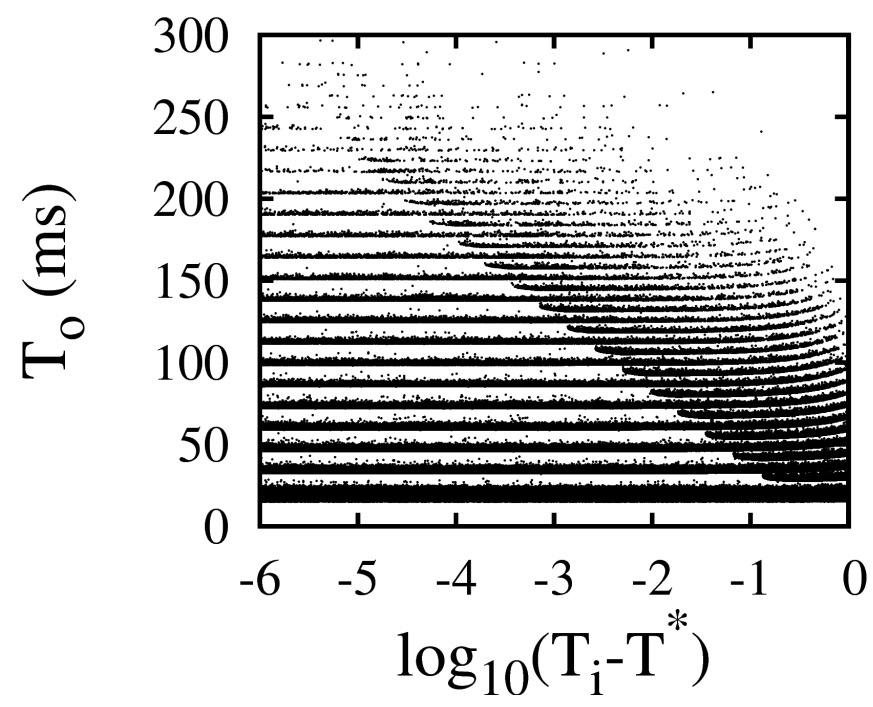

FIG. 6: Scaling of the excitation edge of (a) odd-only multiples of the input $T_{i}$, and (b) all integer multiples, in the chaotic region between $k=2$ and $k=3$. For $g=0.2 \mathrm{mS} / \mathrm{cm}^{2}$, the transition occurs at $T=6.54175 \mathrm{~ms}$.

are sequences of diminishing peaks occuring at integer multiples of the input interspike separation. In Fig. 5 the lowest element of the sequence is missing due to the refractoriness of the neuron. Similar form of ISIH was obtained in a theoretical study of a bistable system stimulated by periodic function with additive Gaussian noise,$\stackrel{20}{,}$ where the presence of noise was essential. However the multimodal histogram was also obtained in a simulation of a deterministic modification of the $\mathrm{HH}$ model ${ }^{21}$.

The HH model studied here does not contain stochastic terms. The multimodal response in Fig. 5 is a result of a deterministic nonlinearity. Thus noise is not the only ingredient enabling the reproduction of the multimodal experimental ISIH. It is possible to identify the source of multimodality by studying ISIH in more detail. 


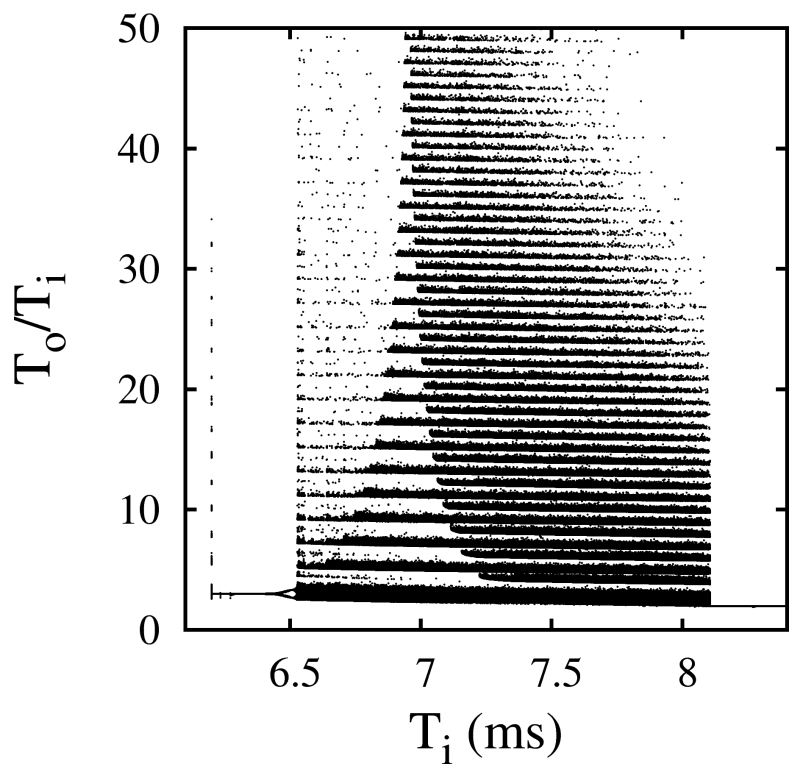

FIG. 7: The multimodal transition at $g=0.17 \mathrm{mS} / \mathrm{cm}^{2}$.

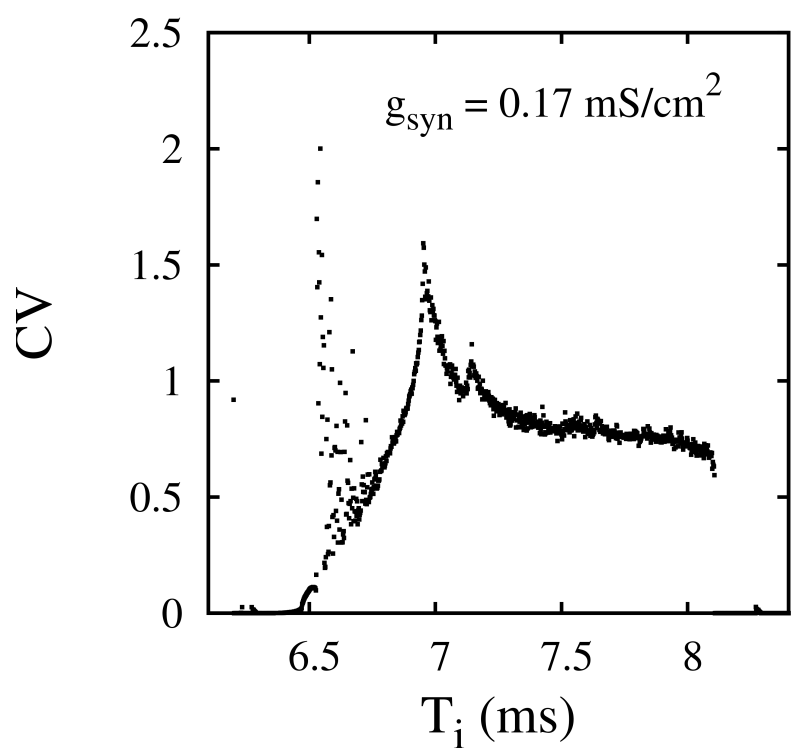

FIG. 8: Coefficient of variation for $g=0.17 \mathrm{mS} / \mathrm{cm}^{2}$. The variability near $T_{i}=6.6 \mathrm{~ms}$ is due to the proximity to the firing threshold.

Close to the excitation threshold, at $g_{\text {syn }} \simeq$ $0.2 \mathrm{mS} / \mathrm{cm}^{2}$, there exists a transition from the odd-only ISIH to ISIH with all integer multiples of $T_{i}$, see Fig. 6. Near the transition the edges of high- $k$ clusters scale logarithmically. The scaling holds both along the $T_{i}$ axis and along the $g_{\text {syn }}$ axis. It can be viewed as a competition between the odd and the even multiples of the driving period.

A clear indication of this "spectral" transition is the singular behavior of the coefficient of variation, see Fig. 8. At the transition $\mathrm{CV}$ is of order 1 . and $k$ is significantly larger than 3 . The maximum $k$ occurs approx-

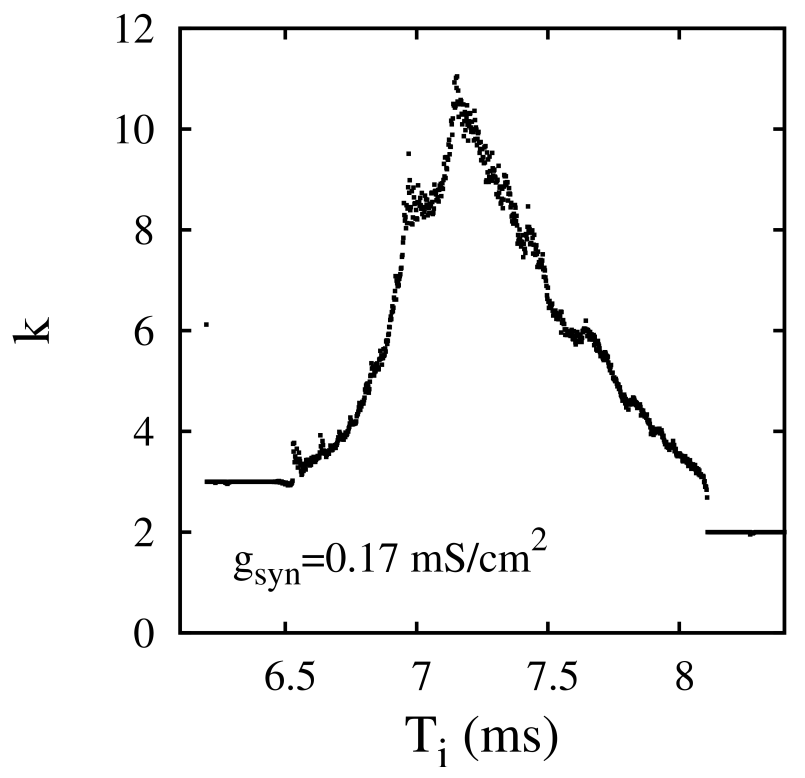

FIG. 9: The ratio $k=T_{o} / T_{i}$ for $g=0.17 \mathrm{mS} / \mathrm{cm}^{2}$. The maximum of $k$ is shifted approximately $0.2 \mathrm{~ms}$ to the right relative to maximum of CV (see Fig. 8).

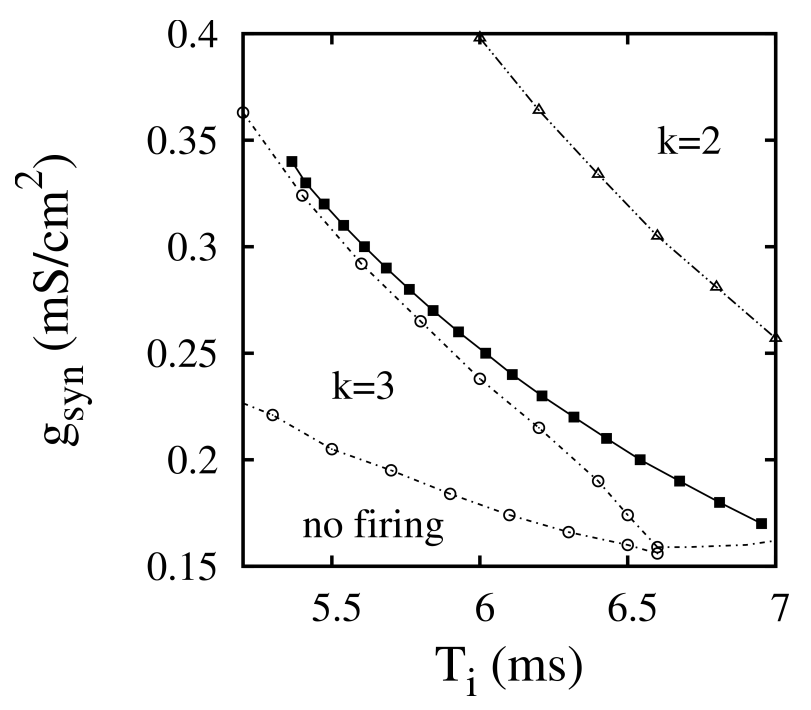

FIG. 10: The location of the multimodal transition (filled squares) on the response diagram.

imately $0.2 \mathrm{~ms}$ above the singularity of $\mathrm{CV}$. One may also think of this shift as a result of relaxation from the constraint of odd-only modes below $T^{*}$. At $T^{*}$ the highest even modes become available and this leads to the increase of $k$.

If such transition were found experimentally it would be a clear sign of the deterministic nonlinear dynamics. In the presence of noise this sharp feature would be smeared and would vanish if noise dominates the dynamics of the system. 


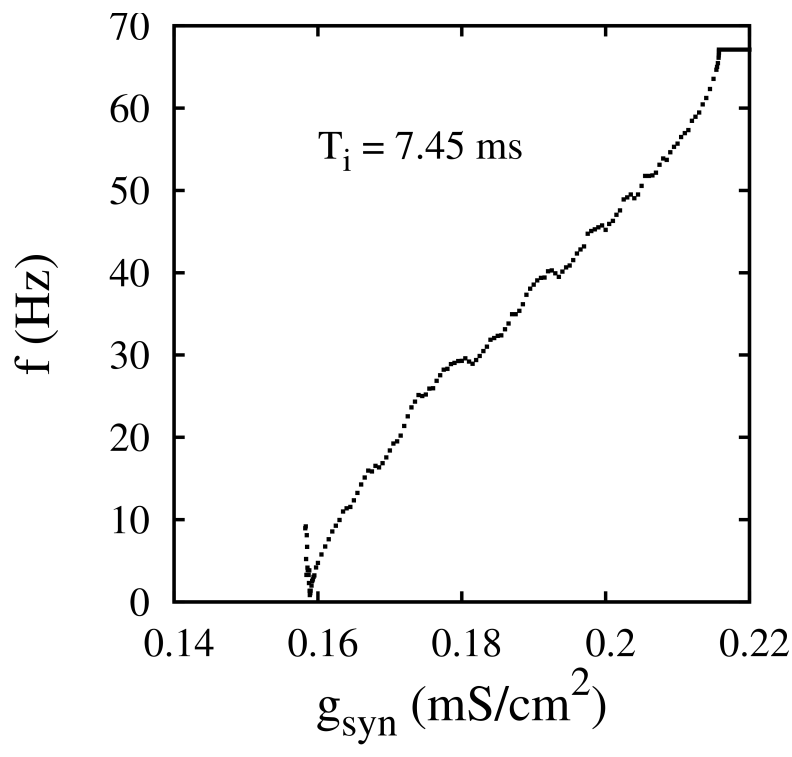

FIG. 11: Frequency vs. synaptic conductivity for $T_{i}=$ $7.45 \mathrm{~ms}$. Each data point is averaged over 15 runs for $60 \mathrm{~s}$ with different initial conditions. The initial $6 \mathrm{~s}$ from each run were discarded.

\section{CONCLUSIONS}

For high synaptic drives in the high frequency regime distinguishing the action potential from the background activity becomes problematic. In this limit the neuron is very sensitive to small changes of the functional form of the signal. For periodic drive with small time constant $\tau$ and $T_{i}$ below $6 \mathrm{~ms}$ the width of the spiking regime along the $g_{s y n}$ axis scales linearly with $T_{i}$. The quality of the neuron's response deteriorates linearly with increasing $g_{\text {syn }}$. This is in contrast to findings for a sinusoidal signal, and more generally for a class of signals satisfying the constraint of charge balancing, where the spiking action remains well defined in the high-frequency limit.

A mechanism of suppression of the neuron's activity might help explain self-regulating behavior of neocortical networks. Various mechanisms of homeostatic action for neural microcircuits were proposed ${ }^{22}$ It would be useful to investigate whether more realistic extensions of the Hodgkin-Huxley model also exhibit self-regulation in response to high-frequency inputs. The network of such neurons would have a "safety switch" built in at the level of individual cells. For $T_{i}$ between 4 and $6 \mathrm{~ms}$ the upper critical synaptic conductivity is of order $2 \mathrm{mS} / \mathrm{cm}^{2}$, which is in the realistic range for neocortical pyramidal neurons 23 .

The input ISI of $4-8 \mathrm{~ms}$ is important to understanding the dynamics of the Hodgkin-Huxley model. In the chaotic region between the $k=2$ and $k=3$ locked states the coefficient of variation of ISI has a singularity at the transition between the odd-only and all-integer multiples of the driving period. The odd modes dominate in the vicinity of the $k=3$ state. The low- $k$ (high- frequency) bands vanish logarithmically near the line of critical points $\left(g_{s y n}, T^{*}\right)$. The firing rate has a minimum at $T_{i} \simeq T^{*}+0.2 \mathrm{~ms}$. Periodically stimulated giant axons of squid have similar nonmonotonic dependence of the firing rate on the current pulse amplitude between the $k=2$ and $k=3$ states $^{24}$. This experiment also showed linear dependence of the firing rate on pulse amplitude near the threshold for $T_{i}>T^{*}$, similarly to Fig. 11. Although the experimental pulses were rectangular, different from the $\alpha(t)$ form with an exponential tail, the qualitative features do not depend much on the precise shape of a pulse. For short pulses the neuron's reaction is determined mainly by the time integral of the stimulus.

The multimodal response occuring in certain sensory neurons may result from noise $e^{20}$ or deterministic nonlinearity 25 . It would be interesting to look for experimental evidence of the odd-all transition. It found, it would be a clear evidence that the neuron dynamics is dominated by nonlinearity, not noise.

The behavior of the model at small $T_{i}$ may be useful to both coincidence detection and estimation of the signal strength. The optimal sensitivity in this case is inversely proportional to frequency.

Our calculation also supports the view expressed by authors of Ref $\frac{9}{}$ that boundaries between various parts of the response diagram are not always clear-cut and may form complicated patterns. This statement also applies to the excitation threshold in the chaotic regime.

In the Hodgkin's classification of intrinsic excitability $\underline{26}$ class 1 neurons maintain firing at arbitrarily low frequencies in response to weak inputs and have continuous frequency-current $(f-I)$ curve. Class 2 neurons fire with certain relatively large frequency, usually of order $40-50 \mathrm{~Hz}$, when stimulus exceeds threshold and have a discontinuous $f-I$ curve. Class 1 and class 2 neurons sometimes are described as integrators and resonators respectively 27 . According to the commonly held view a neuron cannot be an integrator and resonator at the same time. However we showed that the deterministic $\mathrm{HH}$ neuron in a chaotic regime near excitation threshold may oscillate with arbitrarily small frequencies and may perform integration at time scales much longer than the period of its main resonance. The character of the response depends strongly on the functional form of the stimulus and parameters of the model. A recent study showed that the same pyramidal neurons behave as integrators in vitro and resonators in vivo $2 \underline{28}$

The multimodal response of the HH neuron near 140$180 \mathrm{~Hz}$ is not a typical resonance since no particular frequency is preferred. The multiples of the driving frequency alternate chaotically. The average output frequency depends nonmonotonically on the stimulus amplitude. Similar nonmonotonic $f$ vs. $I$ relation was found in periodically stimulated giant axons of squid 24 . Smaller stimuli favor higher multiples of the driving period. Studies of large neuronal networks of various types suggest that there may be a complex interplay between the integrating behavior and the resonant action. ${ }^{22}$ 
The ability to precisely control the nerve cell's potential oscillations is important in constructing devices performing the procedure known as Deep Brain Stimulation ${ }^{29}-31$, which operate at frequencies above $100 \mathrm{~Hz}$. While our model does not satisfy the chargebalancing constraint required in the stimulation of invivo systems, we believe the present study improves our understanding of high-frequency neural oscillators.

\section{Acknowledgments}

The author thanks J. W. Mozrzymas, D. Wójcik, K. Bodova, T. Burwick, and P. Suffczyński for discussions.

Computations were performed in the Computer Center of the Tri-city Academic Computer Network in Gdansk.
1 T. J. Sejnowski, Nature (London) 376, 21 (1995).

2 D. Ferster and N. Spruston, Science 270, 756 (1995).

3 A. L. Hodgkin and A. F. Huxley, J. Physiol. (London) 117, 500 (1952).

4 J. R. Clay, Prog. Biophys. Mol. Biol. 88, 59 (2005).

5 W. Gerstner, Phys. Rev. E 51, 738 (1995).

${ }^{6}$ P. C. Bressloff and S. Coombes, Phys. Rev. Lett. 81, 2168 (1998).

7 J. Rinzel and R. Miller, Math. Biosci. 49, 27 (1980).

8 D. Brown, J. Feng, and S. Freerick, Phys. Rev. Lett. 82, 4731 (1999).

9 J. Guckenheimer and R. O. Oliva, SIAM J. Appl. Dyn. Sys. 1, 105 (2002).

10 S. G. Lee and S. Kim, Phys. Rev. E 60, 826 (1999).

11 E. V. Pankratova, A. V. Polovinkin, and E. Mosekilde, Eur. Phys. J. B 45, 391 (2005).

12 S. Luccioli, T. Kreuz, and A. Torcini, Phys. Rev. E 73, 041902 (2006).

13 S. G. Lee and S. Kim, Phys. Rev. E 73, 041924 (2006).

14 D. T. W. Chik, Y. Wang, and Z. D. Wang, Phys. Rev. E 64, 021913 (2001).

15 Y.-Q. Che, J. Wand, W.-J. Si, and X.-Y. Fei, Chaos, Solitons and Fractals 39, 454 (2009).

16 J. R. Engelbrecht and R. Mirollo, Phys. Rev. E 79, 021904 (2009).
17 H. Hasegawa, Phys. Rev. E 61, 718 (2000).

18 J. E. Rose, J. F. Brugge, D. J. Anderson, and J. E. Hind, J. Neurophysiol. 30, 769 (1967).

19 R. M. Siegel, Physica 42D, 385 (1990).

20 A. Longtin, A. Bulsara, and F. Moss, Phys. Rev. Lett. 67, 656 (1991).

21 J. R. Clay, J. Comput. Neurosci. 15, 43 (2003).

22 R. C. Muresan and C. Savin, J. Neurophysiol. 97, 1911 (2007).

23 N. Ho and A. Destexhe, J. Neurophysiol. 84, 1488 (2000).

${ }^{24}$ N. Takahashi, Y. Hanyu, T. Musha, R. Kubo, and G. Matsumoto, Physica D 43, 318 (1990).

25 D. T. Kaplan, J. R. Clay, T. Manning, L. Glass, M. R. Guevara, and A. Shrier, Phys. Rev. Lett. 76, 4074 (1996).

26 A. L. Hodgkin, J. Physiol. 107, 165 (1948).

27 E. M. Izhikevich, Int. J. Bif. Chaos 10, 1171 (2000).

28 S. A. Prescott, S. Ratté, Y. D. Koninck, and T. J. Sejnowski, J. Neurophysiol. 100, 3030 (2008).

29 A. L. Benabid, P. Pollak, C. Gervason, D. Hoffmann, D. M. Gao, M. Hommel, J. E. Perret, and J. de Rougemont, Lancet 337, 403 (1991).

30 R. E. Gross and A. M. Lozano, Neurol. Res. 22, 247 (2000).

31 C. C. McIntyre, M. Savasta, B. L. Walter, and J. L. Vitek, J. Clin. Neurophysiol. 21, 1 (2004). 